\title{
SAX J1712.6-3739: a persistent hard X-ray source as monitored with INTEGRAL ^
}

\author{
M. Fiocchi ${ }^{1}$, A. Bazzano ${ }^{1}$, P. Ubertini ${ }^{1}$, and G. De Cesare ${ }^{1,2,3}$ \\ 1 Istituto di Astrofisica Spaziale e Fisica Cosmica di Roma (INAF), via Fosso del Cavaliere 100, Roma 00133, Italy \\ e-mail: mariateresa.fiocchi@iasf-roma.inaf.it \\ 2 Dipartimento di Astronomia, Universita' degli Studi di Bologna, via Ranzani 1, 40127 Bologna, Italy \\ 3 Centre d'Étude Spatiale des Rayonnements, CNRS/UPS, BP 4346, 31028 Toulouse Cedex 4, France
}

Received 27 April 2007 / Accepted 9 October 2007

ABSTRACT

\begin{abstract}
Context. The X-ray source SAX J1712.6-3739 is a very weak low mass X-ray binary discovered in 1999 with BeppoSAX and located in the Galactic Center. This region has been intensively investigated by the INTEGRAL satellite with an unprecedented exposure time, providing an unique opportunity to study the hard X-ray behavior also for weak objects.

Aims. We analyzed all available INTEGRAL public and private Key-Program observations with the main aim of studying the longterm behavior of this Galactic bulge X-ray burster.

Methods. The spectral results are based on the systematic analysis of all INTEGRAL observations covering the source position performed between February 2003 and October 2006. SAX J1712.6-3739 did not show any flux variation during this period as well as compared to previous BeppoSAX observations. Hence, to better constrain the physical parameters we combined data from both instruments.

Results. Long INTEGRAL monitoring reveals that this X-ray burster is a weak persistent source, displaying an X-ray spectrum extended to high energy and being most of the time in a low luminosity hard state. The broad-band spectrum is well modeled with a simple Comptonized model with a seed photon temperature of $\sim 0.5 \mathrm{keV}$ and an electron temperature of $\sim 24 \mathrm{keV}$. The low mass accretion rate $\left(\sim 2 \times 10^{-10} M_{\text {sun }} / \mathrm{yr}\right)$, the long bursts recurrence time, the small sizes of the region emitting the seed photons consisting of the inner disk radius and the high luminosity ratio in the $40-100 \mathrm{keV}$ and $20-40 \mathrm{keV}$ band, are all features common to the Ultra Compact source class.

Conclusions. We report, for the first time, on the X-ray behavior of this source: observations with unprecedent wide energy band and sensitivity revealed this X-ray burster is a persistent source with a hard spectrum extending up to energies $>100 \mathrm{keV}$.
\end{abstract}

Key words. gamma rays: observations - stars: individual: SAX J1712.65-3739 - stars: neutron - X-rays: binaries

\section{Introduction}

SAX J1712.6-3739 was discovered in 1999 during the monitoring campaign of the Galactic Center region performed by the BeppoSAX/WFC (Cocchi et al. 1999; in 't Zand et al. 1999). This instrument observed a type-I X-ray burst with exponential decays on August 1999, identifying the compact object as a weakly magnetized neutron star in a low mass X-ray binary system with a derived distance of $7 \mathrm{kpc}$ (Cocchi et al. 2001). The source was followed-up by the BeppoSAX/instruments to investigate the spectral properties: SAX J1712.6-3739 shows a spectrum extended up to about $60 \mathrm{keV}$, with an intensity of $\sim 6 \mathrm{mCrab}$, both in the MECS $(1-10 \mathrm{keV})$ and in the PDS (15-60 keV). The spectrum was fitted by an absorbed power law $(\Gamma \sim 2.2)$ and a column density $N_{\mathrm{H}} \sim 2 \times 10^{22} \mathrm{~cm}^{-2}$ or by a Comptonized emission model with electron temperature of $\sim 50 \mathrm{keV}$ and a lower column density $N_{\mathrm{H}} \sim 1 \times 10^{22} \mathrm{~cm}^{-2}$ (Cocchi et al. 2001). The ROSAT source J171237.1-373834 is just 0' 6 from the BeppoSAX position with a flux of $1.6 \mathrm{mCrab}$ in the energy range $0.1-2.4 \mathrm{keV}$ (in 't Zand et al. 1999). No optical counterpart has been identified yet within the $13^{\prime \prime}(1 \sigma)$ ROSAT

* INTEGRAL is an ESA project with instruments and Science Data Centre funded by ESA member states (especially the PI countries: Denmark, France, Germany, Italy, Switzerland, Spain), Czech Republic and Poland, and with the participation of Russia and the USA. error circle radius. SAX J1712.6-3739, monitored during the PCA/RXTE bulge scan program, is continuously active, apparently in two states: a slowly changing state, and a quicker one (in 't Zand et al. 2007). It was included in the IBIS/ISGRI soft gamma-ray survey catalog (Bird et al. 2007) as a transient X-ray buster with fluxes corresponding to $4.7 \pm 0.1 \mathrm{mCrab}$ and $4.0 \pm$ $0.2 \mathrm{mCrab}$ in the energy bands $20-40 \mathrm{keV}$ and $40-100 \mathrm{keV}$, respectively. Recently, Chelovekov et al. (2006) reported two further burst detections with INTEGRAL/IBIS in 2003-2004, both with the same peak flux.

\section{Observations and data analysis}

BeppoSAX observed the source on September 1999 with an exposure of $21 \mathrm{ks}$. LECS, MECS and PDS event files and spectra, available from the ASI Scientific Data Center, were generated by means of the Supervised Standard Science Analysis (Fiore et al. 1999). Both LECS and MECS spectra were accumulated in circular regions of $8^{\prime}$ radius. The PDS spectra were obtained with the background rejection method based on fixed rise time thresholds. Publicly available matrices were used for all the instruments. The cross-calibration constant values were taken in agreement with the indications given in Fiore et al. (1999). Fits are performed in the following energy band: $0.5-3.0 \mathrm{keV}$ for LECS, 1.5-10.0 keV for MECS and 15-70 keV for PDS. 
Table 1. Results of the SAX J1712.6-3739 fit, using different models: a simple Comptonized model, a blackbody plus a Comptonized component and a multicolor disk blackbody plus a Comptonized component. Uncertainties are at the $90 \%$ confidence level for a single parameter variation.

\begin{tabular}{|c|c|c|c|c|c|c|c|c|}
\hline $\begin{array}{l}\text { Model } 1 \\
\text { comptt }\end{array}$ & $\begin{array}{c}N_{\mathrm{H}} \\
\left(10^{22} \mathrm{~cm}^{-2}\right) \\
1.3 \pm 0.1\end{array}$ & $\begin{array}{l}\cdots \\
\cdots \\
\ldots\end{array}$ & $\begin{array}{l}\cdots \\
\ldots \\
\ldots\end{array}$ & $\begin{array}{c}T_{\mathrm{o}} \\
(\mathrm{keV}) \\
0.47 \pm 0.02\end{array}$ & $\begin{array}{c}T_{\mathrm{e}} \\
(\mathrm{keV}) \\
24_{-10}^{+16}\end{array}$ & $\begin{array}{c}\tau \\
\cdots \\
2.7 \pm 1.3\end{array}$ & $\begin{array}{c}n_{\text {COMPTT }} \\
\left(10^{-3}\right) \\
2.0_{-0.8}^{+1.2}\end{array}$ & $\begin{array}{c}\chi^{2} / \text { d.o.f } \\
\ldots \\
142 / 134\end{array}$ \\
\hline Model 2 & $\begin{array}{c}N_{\mathrm{H}} \\
\left(10^{22} \mathrm{~cm}^{-2}\right)\end{array}$ & $\begin{array}{c}T_{\text {in }} \\
(\mathrm{keV})\end{array}$ & $\begin{array}{c}r_{\text {in }}(\operatorname{cosi})^{0.5} \\
(\mathrm{~km})\end{array}$ & $\begin{array}{c}T_{\mathrm{o}} \\
(\mathrm{keV})\end{array}$ & $\begin{array}{c}T_{\mathrm{e}} \\
(\mathrm{keV})\end{array}$ & $\tau$ & $\begin{array}{c}n_{\text {COMPTT }} \\
\left(10^{-3}\right)\end{array}$ & $\chi^{2} /$ d.o.f \\
\hline$d i s k b b+c o m p t t$ & $1.4 \pm 0.3$ & $0.7 \pm 0.3$ & $<11$ & $0.5 \pm 0.2$ & $24_{-9}^{+22}$ & $3 \pm 2$ & $1.6_{-0.8}^{+1.6}$ & $120 / 132$ \\
\hline Model 3 & $\begin{array}{c}N_{\mathrm{H}} \\
\left(10^{22} \mathrm{~cm}^{-2}\right)\end{array}$ & $\begin{array}{c}T_{\mathrm{bb}} \\
(\mathrm{keV})\end{array}$ & $\begin{array}{c}R_{\mathrm{bb}} \\
(\mathrm{km})\end{array}$ & $\begin{array}{c}T_{\mathrm{o}} \\
(\mathrm{keV})\end{array}$ & $\begin{array}{c}T_{\mathrm{e}} \\
(\mathrm{keV})\end{array}$ & $\begin{array}{l}\tau \\
\ldots\end{array}$ & $\begin{array}{c}n_{\text {COMPTT }} \\
\left(10^{-3}\right)\end{array}$ & $\chi^{2} /$ d.o.f \\
\hline bbody + comptt & $1.2 \pm 0.2$ & $0.6 \pm 0.1$ & $5 \pm 1$ & $1.2 \pm 0.2$ & $34_{-14}^{+54}$ & $2.0 \pm 1.5$ & $0.5_{-0.2}^{+0.6}$ & $119 / 132$ \\
\hline
\end{tabular}

The INTEGRAL (Winkler et al. 2003) observations are divided into uninterrupted 2000 -s intervals, the so-called science windows (SCWs). Wideband spectra (from 5 to $200 \mathrm{keV}$ ) of the source are obtained using data from the two high-energy instruments JEM-X (Lund et al. 2003) and IBIS (Ubertini et al. 2003). Data were processed using the Off-line Scientific Analysis (OSA v5.1 and v6.0 for IBIS and JEM-X, respectively) software released by the INTEGRAL Scientific Data Centre. While IBIS provide a very large FOV $\left(>30^{\circ}\right)$, JEM-X has a narrower FOV $\left(>10^{\circ}\right)$, thus providing only a partial overlap with the highenergy detectors. Data from the Fully Coded field of view only have been used for both instrument. Two X-ray bursts detected with INTEGRAL/IBIS in 2003-2004 reported by Chelovekov et al. (2006) are not included in our data set, being out side our selected field of view. IBIS light curves and spectra are extracted for each individual SCW. JEM-X spectrum was extracted from mosaic image at the position of the SAX J1712.6-3739.

\section{Spectral analysis of the persistent emission}

During the BeppoSAX observations the source intensity was $\sim 6 \mathrm{mCrab}$ both in the MECS $(2-10 \mathrm{keV})$ and PDS (15-60 keV) instruments. The SAX J1712.6-3739 flux level after discovery was near the limiting sensitivity of the WFC instrument (Jager et al. 1997) and for this reason this object was classified as a transient source (Cocchi et al. 1999). INTEGRAL long monitoring, at the limiting sensitivity of $\sim 1 \mathrm{mCrab}$ at $10 \sigma$ level on an exposure of $\sim 10 \mathrm{Ms}$, shows that SAX J1712.6-3739 is a weak persistent source, with a time averaged intensity of $6.1 \pm 0.3 \mathrm{mCrab}$ and $6.7 \pm 0.7 \mathrm{mCrab}$, for IBIS $(17-30 \mathrm{keV})$ and JEM-X $(4-10 \mathrm{keV})$ instruments, respectively. The SAX J1712.6-3739 luminosities do not significantly vary from one observation to another, so we can take advantages in order: 1) to create a high quality IBIS and JEM-X average spectra with long exposure time $(685 \mathrm{ks}$ and $132 \mathrm{ks}$, respectively) and 2) to build up a wide band spectrum with no gap from 0.5 to $200 \mathrm{keV}$, combining LECS, MECS, PDS, IBIS and JEM-X data.

The whole data set was fitted with several physical models, using XSPEC v. 11.3.1.

We fitted the spectrum in the energy band $0.5-200 \mathrm{keV}$ with a simple cutoff powerlaw model but this resulted in a poor fit to the data with a reduced chi square $\chi_{v}^{2} \sim 2.7$ (135 d.o.f.). Similar result $\left(\chi_{v}^{2} \sim 3.1\right.$ with 135 d.o.f.) was obtained fitting this data set with the Comptonization model compsT (Sunyaev \& Titarchuk 1980).

For both models, the strongest residuals are at the energy below a few $\mathrm{keV}$, indicating that a more complex model with a soft component is needed to fit these data. This is in agreement with the picture formulated in recent years according to which the X-ray spectra of the low mass X-ray binaries were generally described as the sum of a soft and hard component. These in turn are interpreted in the framework of two models: the Eastern Model (Mitsuda et al. 1984) consisting of a blackbody together with a Comptonized component and the Western model (White et al. 1984), consisting of a multicolor disk blackbody plus a Comptonized component. For the soft component we used the black body component in the formulation of Mitsuda et al. (1984) with all parameters frees or the multicolor disk blackbody component in the formulation of Makishima et al. (1986), which assumes the gravitational energy released by the accreting material is locally dissipated into blackbody radiation, that the accretion flow is continuous throughout the disk, and finally the effects of electron scattering are negligible. The two parameters of this model are free: $r_{\text {in }}(\cos i)^{0.5}$ where $r_{\text {in }}$ is the innermost radius of the disk, $i$ is the inclination angle of the disk and $k T_{\text {in }}$ the blackbody effective temperature at $r_{\text {in }}$. Adding a black body component improves the fit significantly, reducing $\chi^{2} /$ d.o.f. from $366 / 135$ to $138 / 133$ for the cutoff power law model and from $414 / 135$ to $160 / 133$ for the compsT model. We also have tried to fit this data set with a model consisting of only one COMPTT (Titarchuk 1994) component, assuming a spherical geometry for the Comptonizing region. This replaces the COMPST Comptonization model in the sense that the theory is extended to include relativistic effects and the seed photon energy can be in the soft X-ray range, while in the older models the seed photons are cool, i.e. in the UV band. We left the following parameters free: the temperature of the Comptonizing electrons $k T_{\mathrm{e}}$, the plasma optical depth $\tau_{\mathrm{p}}$ and the input temperature of the soft photon Wien distribution $k T_{0}$. This model gives a good $\chi^{2} /$ d.o.f. of 142/134 and also accounts for the photon deficit below a few $\mathrm{keV}$ with respect to the cutoff powerlaw or COMPST models. The physical parameters obtained using this model are reported in Table 1. Adding a soft X-ray component to the cOMPTT component reduces $\chi^{2}$ /d.o.f. from $142 / 134$ to $119 / 132$ for the black body component and from 142/134 to 120/132 for multicolor disk blackbody component, with a low F-test chance probabilities of $8.6 \times 10^{-6}$ and $1.5 \times 10^{-5}$, respectively. These fit results of the broad band spectrum using the models with two emission components are reported in Table 1.

The column density $N_{\mathrm{H}}$ towards the source was left free and its value measured by the LECS and MECS instrument is always close to the Galactic column density $\left(N_{\mathrm{H}}\right.$ galactic $=1.34 \times$ $10^{22} \mathrm{~cm}^{-2}$, estimated from the $21 \mathrm{~cm}$ measurement of Dickey \& Lockman 1990). Figures 1-3 show the counts spectra with residuals in respect of the best fits and the photon spectra of the SAX J1712.6-3739 using the models described above. These figures clearly illustrate the importance of the broad band coverage obtained combining INTEGRAL and BeppoSAX data, which 

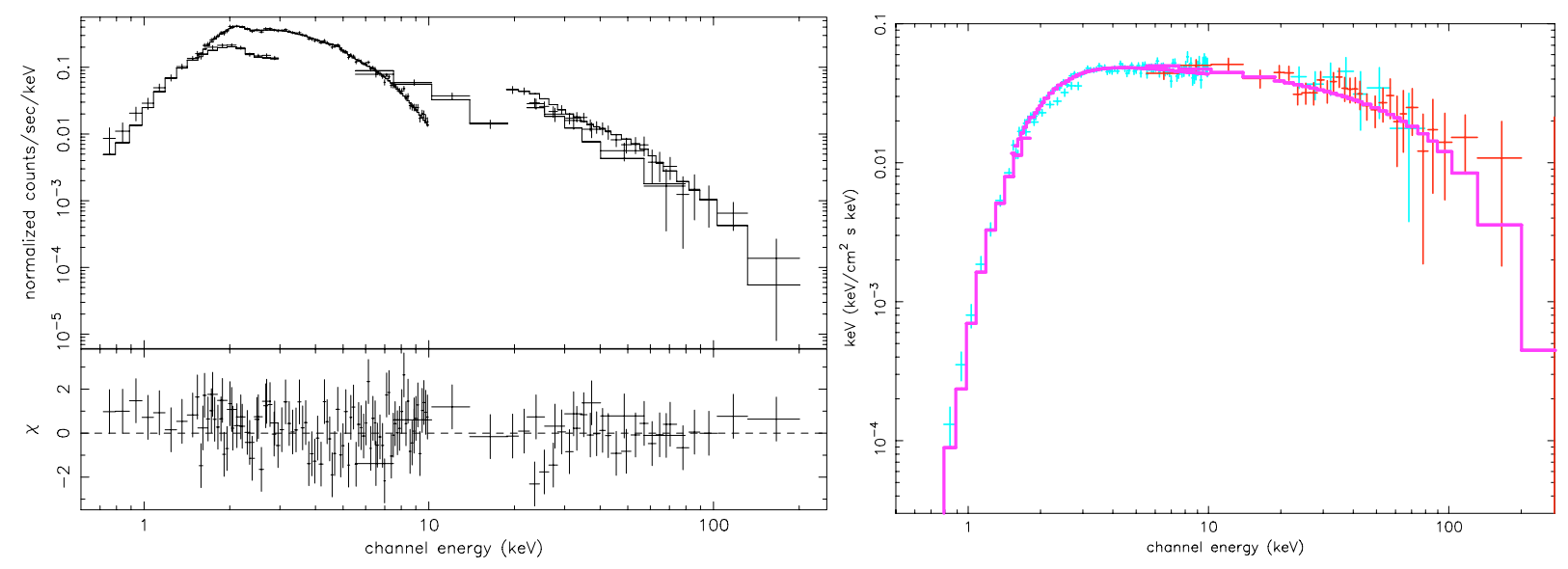

Fig. 1. INTEGRAL and BeppoSAX data and residuals with respect to the best model consisting of a simple Comptonized cOMPTT model (left) and respective energy spectrum (right). Light blue points are BeppoSAX data and red points are INTEGRAL.
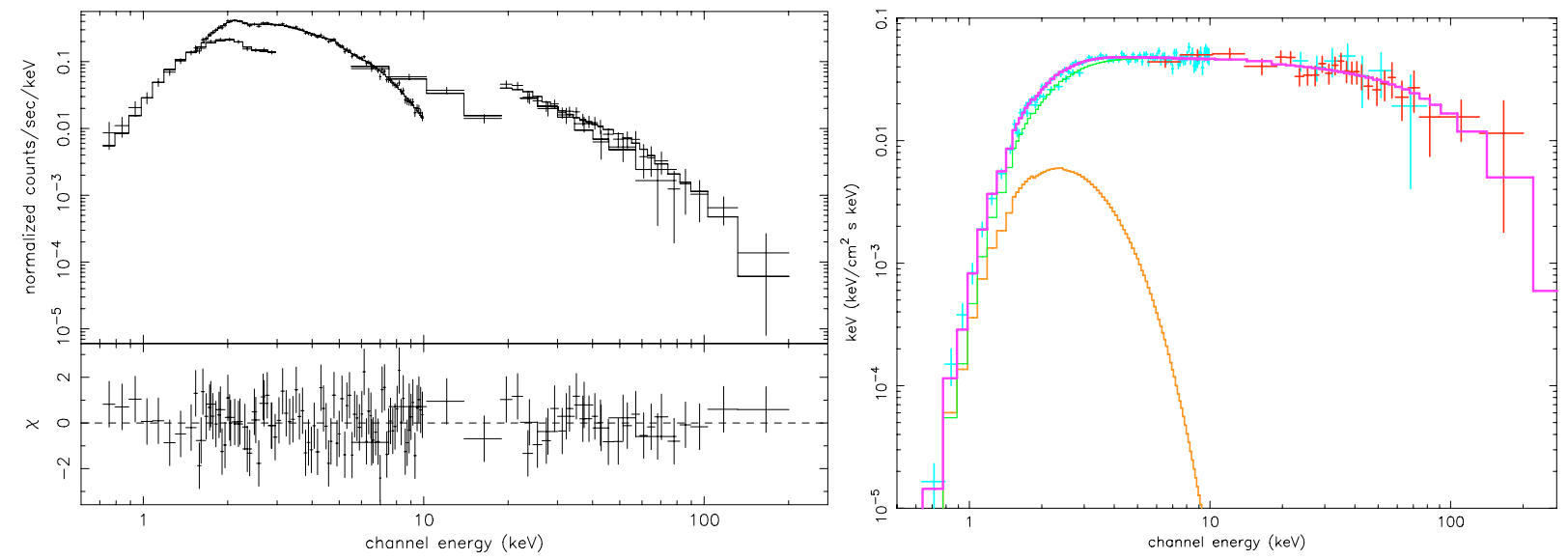

Fig. 2. INTEGRAL and BeppoSAX data and residuals with respect to the best model consisting of a multicolor black body and Comptonized COMPTT model (left) and respective energy spectrum (right). Light blue points are BeppoSAX data and red points are INTEGRAL. The contributions of the two component are shown separately: orange and green are multicolor black body and Comptonization component, respectively, and their sum is plotted in magenta.

allowed us to properly constrain the physical parameters and clearly shows the source has a constant luminosity from $\sim 3$ up to $20 \mathrm{keV}$.

\section{Discussion}

INTEGRAL observations reveal for the first time that this $\mathrm{X}$-ray burster is a persistent source, displaying a very hard X-ray spectrum and spending most of the time in a low luminosity and hard state. A broad-band spectrum obtained combining the INTEGRAL together with the BeppoSAX data displays properties which fit well into the classification of low luminosity $\left(\sim 0.01 L_{\text {Edd }}\right)$ weakly magnetized neutron stars with hard spectra (Barret et al. 2001): a broad-band spectrum extending up to $100 \mathrm{keV}$, dominated by a Comptonized component, with an optical depth of the Comptonizing corona of $\sim 3$, seed photons temperature of $\sim 0.5 \mathrm{keV}$, an electron temperature of $\sim 24 \mathrm{keV}$. A few LMXBs appear to spend most of the time in this hard state (Di Salvo \& Stella 2002), e.g. 4U 0614+091 (Piraino et al. 1999) or 4U1850-087 (Sidoli et al. 2006).

The emission spectrum could also be fitted with a two component model consisting of a blackbody, or multi color blackbody component which represents emission from an optically thick accretion disk or from the neutron star surface, together with a Comptonized component which is interpreted as emission from a hot inner disk region or a boundary layer between the disk and the neutron star. We have estimated a black body radius of $\sim 5 \mathrm{~km}$ for the black body component and the inner radius of the disk $<11 \mathrm{~km}$ for the multi disk component. Although the data statistics do not allow us to distinguish between the two physical models, both values of the radius give an indication of the system compactness.

We have also estimated the sizes of the emitting seed photons region, assuming their emission as blackbody with temperature $T_{0}$ and radius $R_{\text {seed }}$ following in 't Zand et al. (1999). We obtain $R_{\text {seed }} \simeq 8.3 \mathrm{~km}\left(L_{\mathrm{C}} / 10^{37} \mathrm{erg} \mathrm{s}^{-1}\right)^{1 / 2}(1+y)^{-1 / 2}\left(k T_{0} / 1 \mathrm{keV}\right)^{-2}$, where $L_{\mathrm{C}}$ is the luminosity of the Comptonization component. Here, we have estimated the amplification factor of the seed luminosity by the Comptonization as $(1+y)$, where $y$ is the Comptonization parameter, $y=4 k T_{\mathrm{e}} \max \left(\tau, \tau^{2}\right) / m_{\mathrm{e}} c^{2}$. The obtained values are $\sim 1 \mathrm{~km}$ and $\sim 10 \mathrm{~km}$ for the model with the blackbody component and with multicolor disk blackbody component, respectively. The parameters obtained using the latter model allow for a more reasonable interpretation.

These results show that the radius of the region providing the seed photons for the Comptonization emission is consistent with the inner radius of the accretion disk, showing that the seed photons are bounded in a small region spatially coincident with 

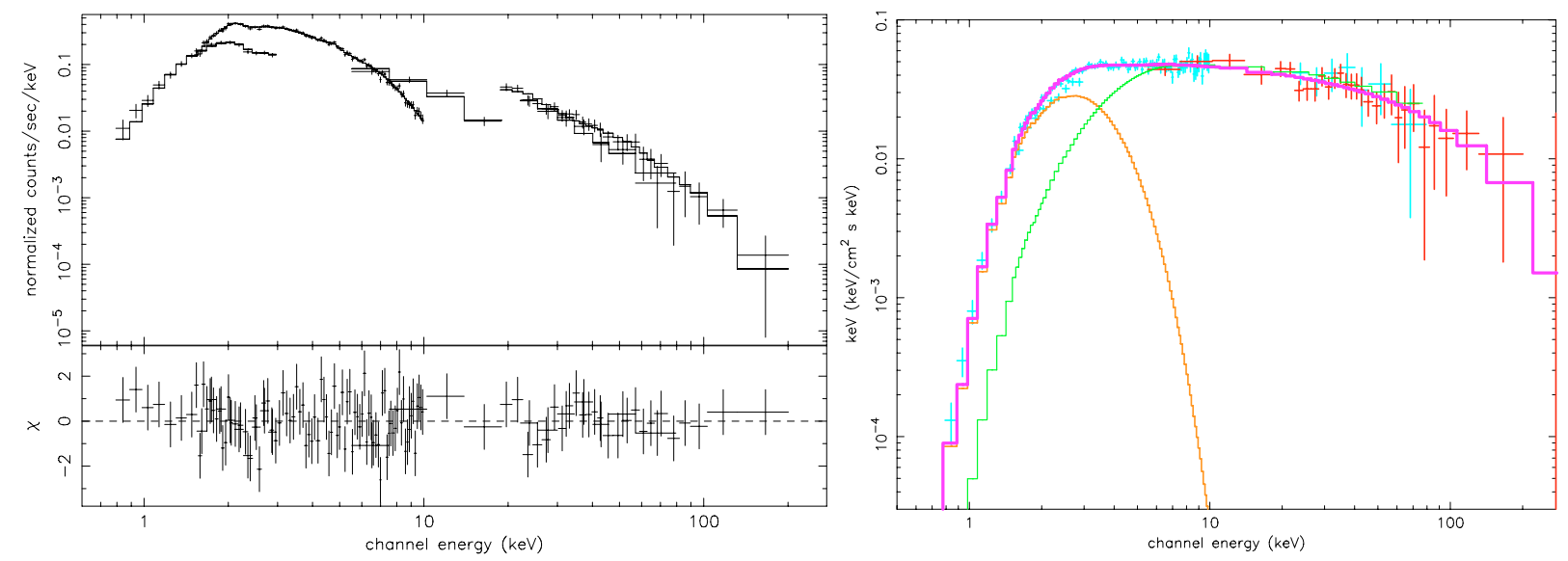

Fig. 3. INTEGRAL and BeppoSAX data and residuals with respect to the best model consisting of a simple black body and a Comptonized COMPTT model (left) and respective energy spectrum (right). Light blue points are BeppoSAX data and red points are INTEGRAL. The contributions of the two component are shown separately: orange and green are the black body and Comptonization component, respectively, and their sum is plotted in magenta.

the inner part of the disk. Moreover the seed photons temperature $T_{0}$ is consistent with the inner disk temperature $T_{\text {in }}$. By imposing $T_{0}=T_{\text {in }}$, the fit values correspond to a disk temperature of $0.57 \pm 0.08 \mathrm{keV}$ with a $\chi^{2} /$ d.o.f. of $121 / 133$. This behavior suggests that the inner disk can be identified as the region providing the seed photons of the Comptonization emission. This result was observed in most of ultra compact X-ray sources: 4U 1850-087, 4U 1820-303 and 4U 0513-40 (Sidoli et al. 2001), XTE J1751-305 (Gierliński \& Poutanen 2005), XTE J1807-294 (Falanga et al. 2005).

Assuming the persistent flux represents the average mass accretion rate and an accretion efficiency of $\eta=0.2$ (corresponding, e.g., to $M_{\mathrm{NS}}=1.4 M_{\odot}$ and $R_{\mathrm{NS}}=10 \mathrm{~km}$ ) and using multi color disk model luminosity, $L_{0.1-200 \mathrm{keV}}=1.6 \times 10^{36} \mathrm{erg} \mathrm{s}^{-1}$, we get $\dot{M} \simeq 2.8 \times 10^{-10} M_{\odot} \mathrm{yr}^{-1}$ for SAX J1712.6-3739. This low mass accretion rate is consistent with the hard spectrum of SAX J1712.6-3739, according to our present understanding on the X-ray bursters (for review see van der Klis 2006). Moreover, the burst recurrence time is dependent on the mass accretion rate on the neutron star: the faster new fuel is provided from the donor star, the shorter is the burst recurrence time. Long INTEGRAL monitoring shows clearly that the frequency of bursts in this source is low, as previously reported by in 't Zand et al. (2007), who estimated a recurrence time of 345-6507 h.

In 't Zand et al. (2007), the authors have selected all 31 persistent X-ray bursters as reported in the INTEGRAL survey (Bird et al. 2006) and demonstrated that the low mass accretion rates are accompanied by the hard X-ray spectra of the persistent emission. Almost all the Ultra Compact X-ray binaries have the highest values of the (40-100) $\mathrm{keV} /(20-40) \mathrm{keV}$ hardness ratio. SAX J1712.6-3739 was not included as it is supposed to have a transient nature. We now know it is a persistent and weak source, showing a high ratio $L_{(40-100) \mathrm{keV}} / L_{(20-40) \mathrm{keV}} \sim 0.90$, in agreement with the value of $0.85 \pm 0.06$ as derived from recent data in Bird et al. (2007). Finally, the low mass accretion rate, the long bursts recurrence time, the small sizes of the region emitting the seed photons consisting with the inner disk radius and the high luminosity ratio in the $40-100 \mathrm{keV}$ and $20-40 \mathrm{keV}$ support the idea of in 't Zand et al. (2007) that this source could be a candidate Ultra Compact Binary.

Acknowledgements. We are grateful to Steven N. Shore for his interest in and support of this work. We acknowledge the ASI financial/programmatic support via contracts ASI-IR I/023/05/0. A special thank to M. Federici for supervising the INTEGRAL data archive and to in 't Zand for useful scientific discussions and suggestions.

\section{References}

Barret, D. 2001, in Adv. Space Res., 28, 307

Bird, A. J., Barlow, E. J., Bassani, L., et al. 2006, ApJ, 636, 765

Bird, A. J., et al. 2007, ApJS, in press

Chelovekov, I. V., Grebenev, S. A., \& Sunyaev, R. A. 2006, AstL, 32, 456 [arXiv: astro-ph/0605638]

Cocchi, M., Natalucci, L., in 't Zand, J. J. M., et al. 1999, IAUC, 7247

Cocchi, M., Bazzano, A., Natalucci, L., Heise, J., \& in 't Zand, J. J. M. 2001, Mem. S.A.It., 72,757

Dickey, \& Lockman 1990, ARA\&A, 28, 2

Di Salvo, T., \& Stella, L. 2002, proceedings of the XXII Moriond Astrophysics Meeting, The Gamma-Ray Universe (Les Arcs, March 9-16, 2002), ed. A. Goldwurm, D. Neumann, \& J. Tran Thanh Van (Vietnam: The Gioi Publishers) [arXiv: astro-ph/0207219]

Falanga, M., Bonnet-Bidaud, J. M., Poutanen, J., et al. 2005, A\&A, 436, 647

Fiore, F., Guainazzi, M., \& Grandi, P. 1999, Cookbook for BeppoSAX NFI Spectral Analysis, www .asdc.asi.it/bepposax/software/cookbook Gierliński, M., \& Poutanen, J. 2005, MNRAS, 359, 1261

in 't Zand, J. J. M., Heise, J., Bazzano, A., Cocchi, M., \& Smith, M. J. S. 1999, IAUC, 7243

in 't Zand, J. J. M., Markwardt, C. B., Bazzano, A., et al. 2002, A\&A, 390, 597 in 't Zand, J. J. M., Jonker, P. G., \& Markwardt, C. B. 2007, A\&A, 465, 953 Jager, et al. 1997, A\&AS, 125, 557

Lund, N., Budtz-Jørgensen, C., Westergaard, N. J., et al. 2003, A\&A, 411, L231

Makishima, K., Maejima, Y., Mitsuda, K., et al. 1986, ApJ, 285, 712

Mitsuda, K., Inoue, H., Koyama, K., et al. 1984, PASJ, 36, 741

Piraino, S., Santangelo, A., Ford, E. C., \& Kaaret, P. 1999, A\&A, 349, 77

Sidoli, L., Parmar, A. N., Oosterbroek, T., et al. 2001, A\&A, 368, 451

Sidoli, L., Paizis, A., Bazzano, A., \& Mereghetti, S. 2006, A\&A, 460, 229

Sunyaev, R. A., \& Titarchuk, L. G. 1980, A\&A, 86, 121

Titarchuk, L. 1994, ApJ, 434, 570

Ubertini, R., Lebrun, F., di Cocco, G., et al. 2003, A\&A, 411, 131

Van der Klis, M. 2006, in Compact Stellar X-ray Sources, ed. W. H. G. Lewin, \& M. van der Klis (Cambrige, UK: CUP)

White, N. E., Stella, L., \& Parmar, A. N. 1988, ApJ, 324, 363

Winkler, C., Gehrels, N., Schönfelder, V., et al. 2003, A\&A, 411, 349 